\title{
Oil and the macroeconomy: using wavelets to analyze old issues*
}

Luís Aguiar-Conraria†

\author{
Maria Joana Soares:
}

\begin{abstract}
We use (cross) wavelet analysis to decompose the time-frequency effects of oil price changes on the macroeconomy. We argue that the relation between oil prices and industrial production is not clear-cut. There are periods and frequencies where the causality runs from one variable to the other and vice-versa, justifying some instability in the empirical evidence about the macroeconomic effects of oil price shocks. We also show that the volatility of both the inflation rate and the industrial output growth rate started to decrease in the decades of 1950 and 1960.
\end{abstract}

Keywords: Business cycles, oil shocks, wavelets, cross wavelets, wavelet coherency. JEL Classification: E32, Q43, C49.

\footnotetext{
*Luís Aguiar-Conraria acknowledges financial support from Fundação para a Ciência e a Tecnologia, project "Oil shocks and the Macroeconomy: Econometric estimation, economic modeling and policy implications", PTDC/ECO/64750/2006.

†Departamento de Economia, Universidade do Minho, E-mail address: 1faguiar@eeg.uminho.pt \$Departamento de Matemática, Universidade de Minho, E-mail address: jsoares@math.uminho.pt
} 


\section{Introduction}

Hamilton (1983, 1985), Gisser and Goodwin (1986), Aguiar-Conraria and Wen (2007) and others provide empirical evidence that until mid-1980s oil prices were a significant determinant of U.S. economic activity. Although Hooker (1996) argued that the correlation between oil prices and economic activity is much less clear after 1985, more recently, other approaches have confirmed the robustness of previous results. For example, Kilian (2007) looks at historical accounts and industry sources to identify exogenous oil production shortfalls and Cavallo and $\mathrm{Wu}$ (2006) construct an oil shocks measure based on news exogenous to the U.S. economy. Once these identification methods are considered, the basic results obtained for the 1970s and 1980s are replicated.

In the cited works, the analysis is exclusively done in the time-domain. The frequencydomain is left out. However, some interesting relations may exist at different frequencies: oil prices may act like a supply shock at high and medium frequencies, therefore affecting industrial production, while, in the longer run (lower frequencies) it is the industrial production, through a demand effect, that affects oil prices.

To uncover relations at different frequencies, it is common to utilize Fourier analysis. However, under the Fourier transform, the time information is completely lost, being difficult to distinguish transient relations or to identify structural changes. We use wavelets to analyze the impact of oil price changes in two macroeconomic variables: Industrial Production and Inflation. Following Aguiar-Conraria et al. (2008), three tools are utilized: the wavelet power spectrum, wavelet coherency and wavelet phase-difference. We refer the reader to that paper for a detailed exposition of these tools and their properties.

\section{Wavelet analysis}

Wavelet theory was born in mid-1980s (Grossmann and Morlet 1984, Goupillaud et al. 1984). After 1990, the literature rapidly expanded to several disciplines, such as physics or epidemi- 
ology. Interestingly, this technique is infrequently used in Economics. The work of Ramsey and Lampart (1998a and 1998b), and Gençay et al. (2001a and 2001b) is unknown to the majority of the economists. ${ }^{1}$ Probably, wavelets are not more popular among economists, because they have been applied either to analyze individual time-series (Gallegati and Gallegati 2007) or to individually analyze several time-series (one each time), whose decompositions are then studied using traditional time-domain methods (Ramsey and Lampart 1998a and 1998b). Aguiar-Conraria et al. (2008) showed how the cross-wavelet coherency and the phase-difference can be used to directly study the interactions between two time-series at different frequencies and how they evolve over time. While the wavelet power spectrum describes the evolution of the variance of a time-series at the different frequencies, the wavelet coherency can be seen as a localized correlation coefficient in the time-frequency space. The phase-difference gives us information on the delay between the oscillations of two time-series.

\subsection{Continuous Wavelet Transform}

The continuous wavelet transform, with respect to the wavelet $\psi$, is a function $W_{x}(s, \tau)$ defined as:

$$
W_{x}(s, \tau)=\int_{-\infty}^{\infty} x(t) \frac{1}{\sqrt{s}} \psi^{*}\left(\frac{t-\tau}{s}\right) d t
$$

where $^{*}$ denotes conjugation. The parameter $s$ is a scaling factor that controls the length of the wavelet and $\tau$ is a location parameter that indicates where the wavelet is centered. Scaling a wavelet simply means stretching it (if $|s|>1$ ), or compressing it (if $|s|<1$ )

If the wavelet function $\psi(t)$ is complex, the wavelet transform $W_{x}$ will also be complex. The transform can then be divided into the real part $\left(\mathcal{R}\left\{W_{x}\right\}\right)$ and imaginary part $\left(I\left\{W_{x}\right\}\right)$, or amplitude, $\left|W_{x}\right|$, and phase, $\tan ^{-1}\left(\frac{\mathcal{I}\left\{W_{x}\right\}}{\mathcal{R}\left\{W_{x}\right\}}\right)$. The phase of a given time-series $x(t)$ is parameterized in radians, ranging from $-\pi$ to $\pi$. In order to separate the phase and amplitude information of a time series it is important to make use of complex wavelets. Just like with

\footnotetext{
${ }^{1}$ Among the exceptions to this rule, one can point to Connor and Rossiter (2005), Gençay et al. (2005), and Gallegati and Gallegati (2007).For a recent survey of wavelet applications to economic data see Crowley (2007).
} 
the Fourier tranform, under some regularity conditions, we can reconstruct $x(t)$ from its continuous wavelet transform.

\subsection{The Morlet wavelet}

The minimum requirements imposed on a function $\psi(t)$ to qualify for being a mother (admissible or analyzing) wavelet are that $\psi(t)$ is a square integrable function and satisfies the admissibility condition: $\int_{-\infty}^{\infty} \frac{|\Psi(f)|}{|f|} d f<\infty$, where $\Psi(f)$ is the Fourier transform of $\psi$ For most purposes, the admissibility condition is equivalent to requiring $\int_{-\infty}^{\infty} \psi(t) d t=0$. Therefore, $\psi$ has to wiggle up and down the $t$-axis, behaving like a wave, justifying the choice of the term wavelet.

There are several wavelet functions available, such as Morlet, Mexican hat, Daubechies, etc. The choice depends on the particular application one has in mind. We choose a complex wavelet as it yields information on the amplitude and phase, both essential to study synchronism between different time-series.

An important property of a wavelet function is its accuracy. Define the center of the wavelet $\psi$ by $\mu_{t}=\int_{-\infty}^{\infty} t|\psi(t)|^{2} d t$. Consider the variance $\sigma_{t}^{2}=\int_{-\infty}^{\infty}\left(t-\mu_{t}\right)^{2}|\psi(t)|^{2} d t$. Similarly, define the center $\mu_{f}$ and variance $\sigma_{f}$ of the Fourier transform of $\psi$. The interval $\left[\mu_{t}-\sigma_{t}, \mu_{t}+\sigma_{t}\right]$ is the set where $\psi$ attains its "most significant" values whilst the interval $\left[\mu_{f}-\sigma_{f}, \mu_{f}+\sigma_{f}\right]$ plays the same role for $\Psi$. The rectangle $\left[\mu_{t}-\sigma_{t}, \mu_{t}+\sigma_{t}\right] \times$ $\left[\mu_{f}-\sigma_{f}, \mu_{f}+\sigma_{f}\right]$ in the $(t, f)$-plane is the Heisenberg box in the time-frequency plane. We say that $\psi$ is localized around the point $\left(\mu_{t}, \mu_{f}\right)$ of the time-frequency plane with uncertainty given by $\sigma_{t} \sigma_{f}$. In our context, the Heisenberg's principle establishes that $\sigma_{t} \sigma_{f} \geq \frac{1}{4 \pi}$. The Morlet wavelet, $\psi(t)=\pi^{-\frac{1}{4}} \exp \left(i \omega_{0} t\right) \exp \left(-\frac{1}{2} t^{2}\right)$, is a complex valued wavelet with optimal joint time-frequency concentration, in the sense that it reaches the lower bound, $\sigma_{t} \sigma_{f}=\frac{1}{4 \pi}$. Choosing $\omega_{0}=6$, the wavelet scale, $s$, is inversely related to the frequency, $f \approx \frac{1}{s}$, simplifying the interpretation of the wavelet analysis. 


\subsection{The wavelet power spectrum}

Typically one has to deal with a discrete time-series $\left\{x_{n}, n=0, \ldots, N-1\right\}$ of $N$ observations with a uniform time step $\delta t$ and the integral in (1) is then discretized:

$$
W_{m}^{x}(s)=\frac{\delta t}{\sqrt{s}} \sum_{n=0}^{N-1} x_{n} \psi^{*}\left((n-m) \frac{\delta t}{s}\right), \quad m=0,1, \ldots, N-1
$$

Although it is possible to calculate the wavelet transform using the above formula for each value of $s$ and $m$, one can also identify the computation for all the values of $m$ simultaneously as a convolution of two sequences. The standard procedure is to calculate this convolution as a simple product in the Fourier domain, using the Fast Fourier Transform algorithm to go forth and back from time to spectral domain. As with other types of transforms, the CWT applied to a finite length time-series inevitably suffers from border distortions, which increase with $s$. The region in which the transform suffers from these edge effects is called the cone of influence. In this area, the results are unreliable and have to be interpreted carefully.

The wavelet power spectrum is just $\left|W_{n}^{x}\right|^{2}$. The wavelet power spectrum characterizes the distribution of the energy (spectral density) of a time series across the two-dimensional time-scale plane, leading to a time-scale (or time-frequency) representation. We assess the statistical significance of the wavelet power against the null hypotheses that each variable follows an $A R M A(p, q)$ process, with no pre-conditions on $p$ and $q$. Unfortunately, theoretical distributions for wavelet power have only been derived for $A R(0)$ and $A R(1)$ processes, so we have to rely on Monte-Carlo simulations. The simulations are done using the amplitude adjusted Fourier-transformed surrogates proposed by Schreiber and Schmitz (1996).

\subsection{Wavelet coherency and phase difference}

The cross wavelet transform of two time series, $x=\left\{x_{n}\right\}$ and $y=\left\{y_{n}\right\}$, is simply defined as $W_{n}^{x y}=W_{n}^{x} W_{n}^{y *}$. The cross wavelet power is given by $\left|W_{n}^{x y}\right|$. While the wavelet power 
spectrum depicts the variance of a time series, with times of large variance showing large power, the cross-wavelet power of two time series depicts the covariance between these time series at each scale or frequency. Wavelet coherency is ratio of the cross-spectrum to the product of the spectrum of each series, and can be thought of as the local (both in time and frequency) correlation between two time-series:

$$
R_{n}(s)=\frac{\left|S\left(s^{-1} W_{n}^{x y}(s)\right)\right|}{S\left(s^{-1}\left|W_{n}^{x}\right|\right)^{\frac{1}{2}} S\left(s^{-1}\left|W_{n}^{y}\right|\right)^{\frac{1}{2}}},
$$

where $S$ denotes a smoothing operator in both time and scale - see Aguiar-Conraria et al. (2008) for details. Again, theoretical distributions for wavelet coherency have not been derived yet. Therefore, to assess the statistical significance of the estimated wavelet coherency, we follow we follow Schreiber and Schmitz (1996).

We focus on the wavelet coherency, instead of the wavelet cross spectrum for two reasons: (1) the wavelet coherency has the advantage of being normalized by the power spectrum of the two time-series, and (2) Maraun and Kurths (2004) show that the wavelet cross spectrum can show strong peaks even for the realization of independent processes suggesting the possibility of spurious significance tests.

The phase of a given time-series can be viewed as the position in the pseudo-cycle of the series. The phase-difference describes the relative positions of the two time series:

$$
\phi_{x, y}=\tan ^{-1}\left(\frac{\mathcal{I}\left\{W_{n}^{x y}\right\}}{\mathcal{R}\left\{W_{n}^{x y}\right\}}\right), \quad \text { with } \phi_{x, y} \in[-\pi, \pi]
$$

A phase-difference of zero indicates that the time-series move together at the specified frequency. If $\phi_{x, y} \in\left(0, \frac{\pi}{2}\right)$ then the series move in phase, but the time-series $y$ leads $x$. If $\phi_{x, y} \in\left(-\frac{\pi}{2}, 0\right)$ then it is $x$ that is leading. A phase-difference of $\pi$ (or $\left.-\pi\right)$ indicates an antiphase relation. If $\phi_{x, y} \in\left(\frac{\pi}{2}, \pi\right)$ then $x$ is leading. Time-series $y$ is leading if $\phi_{x, y} \in\left(-\pi,-\frac{\pi}{2}\right)$. 

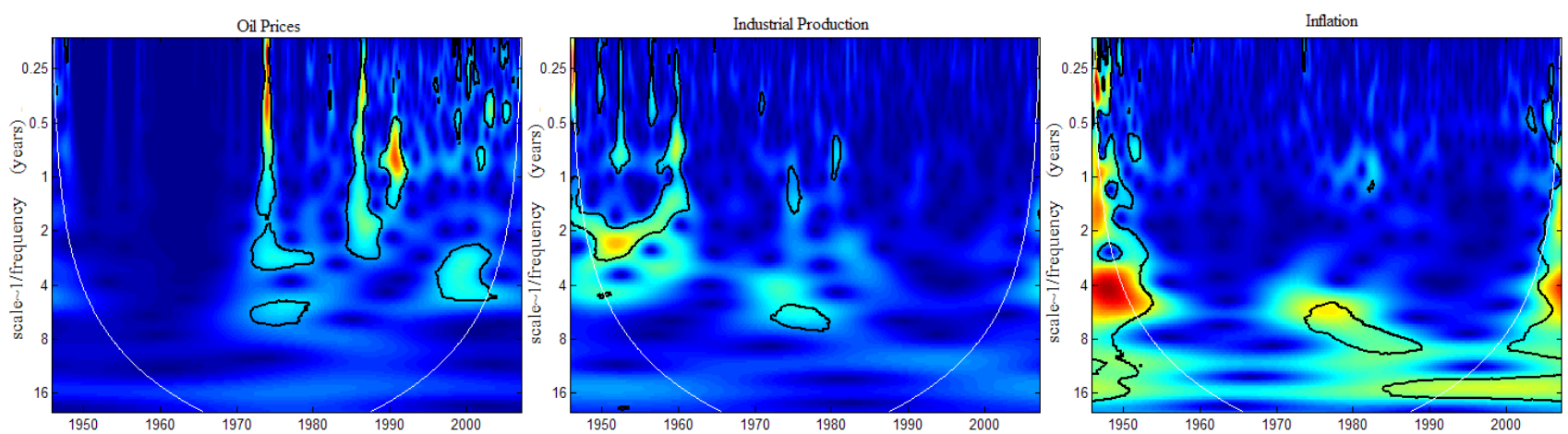

Figure 1: Wavelet Power Spectrum — The black contour designates the $5 \%$ significance. The cone of influence, which indicates the area affected by edge effects, is the outside region of the white line. The color code for power ranges from blue (low power) to red (high power).

\section{Data analysis}

In Figure 1, we can see the estimated power spectrum for several time series for the United States economy: interest rates (3-month Treasury Bills), inflation (based on the Consumer Price Index), Oil Prices (growth rate) and Industrial Production Index (growth rate).

It is clear that the different time series have different characteristics in the time-frequency domain. During the late 1940s and early 1950s, the inflation rate variance was quite high both at low and high scales. In the 1970s and 1980s, probably as a consequence of very active oil shocks, the variance of the inflation rate became higher, but in this case, the effect is clearer at medium and high scales, suggesting that we were facing medium and long term shocks to inflation. The power, at all scales, of the industrial production was quite high until 1950s. After that, it has been steadily decreasing, with an exception between mid 1970s and mid 1980s, when the variance at the business cycle frequency (3 to 8 years) was quite high. It has become common in the literature to argue that we have been observing, in the last two decades, a decrease in the volatility of GDP in the United States (e.g. see Blanchard and Simon 2001). After World War II, the volatility was quite high at business cycle frequencies. In the 1960s, the volatility decreased at all scales. Therefore, we observe that the "Great Moderation" started in the decade of 1960. Then macroeconomic volatility increased again, 

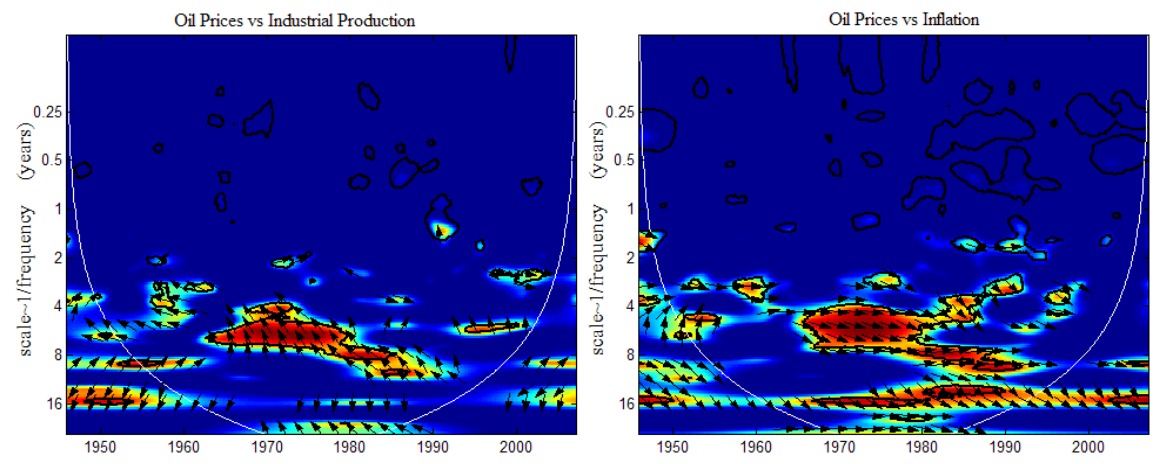

Figure 2: Cross-Wavelet Coherency - The black contour designates the $5 \%$ significance. The cone of influence is the outside region of the white line. The color code for power ranges from blue (low coherency) to red (high coherency). Arrows pointing to the right mean that the variables are in-phase. To the right and up, with oil prices lagging. To the right and down, with oil prices leading. Arrows pointing to the left mean that the variables are out of phase. To the left and up, with oil prices leading. To the left and down, with oil prices lagging.

probably due to the oil shocks, at the business cycle frequency in the 1970s, however this increase was temporary.

If we look at the power spectrum of the Oil Prices growth rate, we observe that until mid-1970s these were very stable. Between 1975 and 1980, both low and medium scales $\frac{1}{12} \sim 6$ years (high and medium frequencies) show high power We observe similar effects in late 1980s and early 1990s, and again in 2000. A structural change occurred in the oil price series in the mid 1970s, after which oil prices became market based and much more volatile.

Figure 2, on the left, gives us the coherency and phase relations between oil prices and industrial production. Several structural changes occurred. In the 1950s, there is high coherency at large scales (in the $10 \sim 16$ years band). ${ }^{2}$ Between mid 1960s and 1990, we can see a high coherency at business cycle frequencies $(3 \sim 8$ years $)$. Looking at the phase difference in the $8 \sim 12$ years band, we can see a positive relation between industrial production and oil prices, with industrial production leading, until mid-1960s. This suggests that in the long run, increases in the industrial production lead to increases in the oil price, suggesting that these oil price increases are demand-driven. If we look at the $3 \sim 8$ years

\footnotetext{
${ }^{2}$ Note that this band is affected by edge effects, so the results should be interpreted conservatively.
} 
band, we see that the phase difference is contained between $\frac{\pi}{2}$ and $\pi$ for most of the time, suggesting an inverse relation between oil prices and industrial production, with oil prices leading. This means that the Industry reacts to increases in the oil prices, and hence in the production costs, decreasing output.

On the right of figure 2 , we see that the relation between oil prices and inflation is even stronger and more stable. The phase differences reveal a very stable relation. At most scales and for most of the time, the phase difference has consistently been between zero and $-\pi / 2$. This suggests that oil price increases lead the consumer price index increases. Looking at coherency some different patterns emerge. There is a structural change in the late 1960s, coinciding with the six-day war of 1967 . Before that time, there were not many periods of high coherency. In the 1970s there is high coherency at both medium ( $4 \sim 8$ years band) and large scales $(12 \sim 16$ years band). During the 1980 decade, we observe high coherency in the $8 \sim 16$ years band After 1990, only at very high scales we observe strong coherency. This suggests that monetary authorities became more proficient on avoiding the inflationary effects of oil price increases. Some political economy major events that happened during these decades may explain this evolution. The decade of 1970 is the decade of major oil shocks. After that, in 1980, there was a strong shift in the American monetary policy. In July 1979, Paul Volcker had been nominated the Chairman of the Federal Reserve Board. Volker announced a fierce fight against inflation and implemented a very restrictive monetary policy as a reaction to the inflationary pressures of the second oil shock. In 1987, and during the entire decade of 1990, when Alan Greenspan was the chairman of the Federal Reserve, inflation was under control.

\section{Conclusion}

Wavelet analysis is an important addition to time-series methods with practical applications in Economics, which allows us to decompose relationships in the time-frequency domain. In 
this paper, wavelets were used to study the evolution of the impact of oil price changes in the macroeconomy.

We have studied the relation between oil and output and uncovered an interesting relation: while at business cycle frequencies (3-8 years) oil prices lead industrial production, with oil price increases having negative effects on production. At lower frequencies and until mid 1960s, production changes lead oil price changes, suggesting that these were demand driven.

The relation between oil price increases and inflation was also studied. This relation proved to be more stable with oil price increases leading inflation increases across all timescales. But an interesting feature was also apparent, the tight monetary policy of the 1980s proved to be successful, with a decrease of the inflationary impact of oil price shocks. During the 1990s, the inflationary impacts of oil price increases was also very well contained.

We have also shown that the volatility of both the inflation rate and the output growth rate started to decrease in the decades of 1950 and 1960, suggesting that the great moderation started then, but that it was temporarily interrupted due to the oils crisis of the 1970s, whose effects were felt until the mid 1980s. 


\section{References}

[1] Aguiar-Conraria, L., Azevedo, N. and Soares, M. J. (2008), Using Wavelets to Decompose the Time-Frequency Effects of Monetary Policy, Physica A: Statistical Mechanics and its Applications, forthcoming, doi: 10.1016/j.physa.2008.01.063.

[2] Aguiar-Conraria, L., and Wen, Y. (2007), Understanding the Large Negative Impact of Oil Shocks, Journal of Money, Credit, and Banking, 39:4, 925-44.

[3] Blanchard, O., and Simon, J. (2001), The Long and Large Decline in U.S. Output Volatility, Brookings Papers on Economic Activity, 1, 135-64.

[4] Cavallo, M., and Wu, T. (2006), Measuring Oil-Price Shocks Using MarketBased Information, Federal Reserve Bank of San Francisco, working paper 2006-28.

[5] Connor, J. and Rossiter, R. (2005), Wavelet Transforms and Commodity Prices, Studies in Nonlinear Dynamics $\&$ Econometrics, 9 (1), Article 6.

[6] Crowley, P. (2007), A Guide to Wavelets for Economists, Journal of Economic Surveys, 21 (2), 207-267.

[7] Gallegati, M. and Gallegati, M. (2007), Wavelet Variance Analysis of Output in G-7 Countries, Studies in Nonlinear Dynamics $\mathcal{E}$ Econometrics, 11 (3), Article 6.

[8] Gisser, M., and Goodwin, T. (1986), Crude Oil and the Macroeconomy: Tests of Some Popular Notions, Journal of Money, Credit, and Banking, 18:1, 95-103.

[9] Gençay, R., Selçuk, F. and Witcher, B. (2001a), Scaling Properties of Foreign Exchange Volatility, Physica A: Statistical Mechanics and its Applications, 289 (12), 249-266.

[10] Gençay, R., Selçuk, F. and Witcher, B. (2001b), Differentiating Intraday Seasonalities Through Wavelet Multi-Scaling, Physica A: Statistical Mechanics and its Applications, 289 (3-4), 543-556. 
[11] Gençay, R., Selçuk, F. and Witcher, B. (2005), Multiscale Systematic Risk, Journal of International Money and Finance, 24, 55-70.

[12] Goupillaud, P., A. Grossman and J. Morlet (1984), Cycle-Octave and Related Transforms in Seismic Signal Analysis, Geoexploration, 23, 85-102.

[13] Grossmann, A. and Morlet, J. (1984), Decomposition of Hardy Functions into Square Integrable Wavelets of Constant Shape, SIAM Journal on Mathematical Analysis, 15, 723-736.

[14] Hamilton, J. (1983), Oil and the Macroeconomy since World War II, Journal of Political Economy, 91:2, 228-48.

[15] Hamilton, J. (1985), Historical Causes of Postwar Oil Shocks and Recessions, The Energy Journal, 6, 97-116.

[16] Hooker, M. (1996), What Happened to the Oil Price-Macroeconomy Relationship?, Journal of Monetary Economics, 38:2, 195-213.

[17] Kilian, L.. (2007), Exogenous Oil Supply Shocks: How Big Are They and How Much Do They Matter for the U.S. Economy?, Review of Economics and Statistics, forthcoming.

[18] Maraun, D. and Kurths, J. (2004), Cross Wavelet Analysis: Significance Testing and Pitfalls, Nonlinear Processes in Geophysics, 11, 505-514.

[19] Ramsey, J. and Lampart, C. (1998a), Decomposition of Economic Relationships by Time Scale Using Wavelets: Money and Income, Macroeconomic Dynamics, $2,49-71$.

[20] Ramsey, J. and Lampart, C. (1998b), The Decomposition of Economic Relationships by Time Scale Using Wavelets: Expenditure and Income, Studies in Nonlinear Dynamics and Econometrics, 3, 23-42. 
[21] Schreiber, T. and Schmitz, A. (1996), Improved Surrogate Data for Nonlinearity Tests, Physical Review Letters, 77:4, 635-638. 